\title{
Cefalea postpunción dural con aguja espinal de bisel cortante vs aguja espinal de punta cónica: revisión sistemática
}

\author{
Postdural puncture headache with cutting spinal needle vs \\ pencil point spinal needle: systematic review
}

Tatiana María Bedoya-Jaramilloª, Elsa María Vásquez Trespalaciosº ${ }^{1 b}$ Mauricio Vasco-Ramírez ${ }^{1 c}$

\begin{abstract}
Introduction: Postdural puncture headache is one of the most frequent complications that occurs after the puncture of the dura mater, resulting in the spinal fluid leakage, through the same and subsequent traction of brain structures. Objective: To compare cutting spinal needle and pencil point spinal needle in the incidence of postural dural puncture headache. Materials and Methods: A literature search of scientific articles published since 2006 was carried out in the Pubmed, Science Direct, Clinical Key and EBSCO databases, which evaluates the incidence of postdural puncture headache in spinal needles with conical tip and cutting bevel, additionally, experts will be consulted in the area that should suggest literature related to this topic, which would not have been included in the search methods previously described. Results: In total, 14 studies were selected in the population that suffered puncture of the dura mater after accidental, therapeutic or diagnostic lumbar puncture (LP) and the presentation of postdural puncture headache was measured according to the ICHD-3 beta criteria. It was found that there is a decrease in the incidence of postdural puncture headache with conical tip spinal needles, compared to spinal needles of cutting bevel. Conclusions: Current evidence indicates that postdural puncture headache occurs less frequently, with low intensity and short duration in patients operated with a conical spinal needle, compared to the cutting bevel. In addition, according to the recently described pathophysiology, it is contradictory that conical spinal needles are called "atraumatic", so this review proposes a change in nomenclature that is most useful for the clinical anesthesiologist.
\end{abstract}

\footnotetext{
Universidad CES, Medellin, Colombia.

Médica general.

Epidemióloga, División de Posgrados Clínicos.

Presidente, Comité de Anestesia Obstétrica, WFSA, Docente facultad de Medicina.
}

Fecha de recepción: 20 de junio de 2019

Fecha de aceptación: 30 de agosto de 2019

\section{ORCID}

https://orcid.org/0000-0002-6865-0478

\section{Correspondencia:}

Mauricio Vasco Ramírez

Jefe de Simulación Clínica,Facultad de Medicina

Email: mvascor@ces.edu.co 


\section{Resumen}

Introducción: La cefalea postpunción dural es una de las complicaciones más frecuentes que se presenta posterior a la punción de la duramadre, lo cual produce salida de líquido cefalorraquídeo, a través de la misma y posterior tracción de las estructuras cerebrales. Objetivo: Comparar la aguja espinal de bisel cortante y la aguja espinal de punta cónica en la incidencia de presentación de cefalea postpunción dural. Materiales y Métodos: Se realizó una búsqueda bibliográfica de artículos científicos publicados desde el año 2006, en las bases de datos Pubmed, Science Direct, Clinical Key y EBSCO, que evaluaran la incidencia de cefalea postpunción dural en agujas espinales de punta cónica y bisel cortante, adicionalmente se consultó con expertos en el área que pudieran sugerir literatura relacionada con este tema, la cual no hubiese sido incluida en los métodos de búsqueda previamente descritos. Resultados: En total se seleccionaron 14 estudios realizados en población que sufrieron punción de la duramadre luego de punción lumbar (PL) accidental, terapéutica o diagnóstica y se midió la presentación de cefalea postpunción dural según los criterios ICHD-3 beta. Se encontró que hay una disminución de la incidencia de cefalea postpunción dural con las agujas espinales de punta cónica, en comparación con las agujas espinales de bisel cortante. Conclusiones: La evidencia actual indica que la cefalea postpunción dural se presenta con menor frecuencia, baja intensidad y corta duración en pacientes intervenidos con aguja espinal de punta cónica, en comparación con la aguja espinal de bisel cortante. Adicionalmente, según la fisiopatologia recientemente descrita, es contradictorio que las agujas espinales de punta cónica sean llamada "atraumáticas", por lo que esta revisión propone un cambio en la nomenclatura que resulta de mayor utilidad para el anestesiólogo clínico.

\section{Palabras clave:}

Cefalea pospunción

dural,

aguja espinal punta de

lápiz,

aguja whitacre, aguja quincke, cefalea

\section{Introducción}

a cefalea postpunción dural (CPPD) es una de las complicaciones más frecuentes e incapacitantes - que se presenta posterior a la punción de la duramadre[1]-[4]. Ocurre en procedimientos que requieren anestesia espinal, punción lumbar (PL) diagnóstica o terapéutica y accidentalmente durante analgesia/ anestesia epidural[1]. Fue descrita de primera vez por el padre de la anestesia espinal August Bier, en agosto de 1898, cuando su asistente le administró anestesia espinal para investigar personalmente los efectos de la técnica, especialmente nauseas, vómito y cefalea; el procedimiento se realizó utilizando aguja punta Quincke de gran calibre, pero el intento fue fallido ya que la jeringa no se ajustó a la aguja ya implantada en el espacio subaracnoideo, horas después presenta cefalea severa, describiendo de Novo un cuadro de CPPD $[5,6]$.

Este cuadro resulta de la salida persistente de líquido cefaloraquídeo (LCR) a través de la duramente, hacia el espacio epidural, que desencadena disminu- ción de la presión intracraneana (PIC) con tracción de las estructuras cerebrales y/o vasodilatación compensatoria de las venas intracraneales bajo la hipótesis de Monroe Kellie[7]-[10].

Es una complicación que sin duda prolonga la estancia hospitalaria, sin embargo, autoresuelve en el $72 \%$ de los pacientes, en caso de no autolimitarse, requiere el uso de intervenciones adicionales que logren detener la fuga de LCR de manera temprana. [11]-[13]. Algunas de las intervenciones que se han descrito para el manejo de la CPPD son, la administración temprana de cafeína, teofilina oral o intravenosa (IV), terapia esteroidea y restauración del volumen de LCR con terapia oral o IV, en caso de que estas medidas fallen, se debe acudir al uso del parche epidural hemático, el cual consiste en la aplicación de sangre en el espacio epidural, alrededor del canal espinal, formando un "parche" que detiene la fuga de LCR, con éxito reportado en un $60-80 \%$ de los casos. [11],[14]-[16].

La CPPD típicamente compromete el área frontal y occipital, se irradia a cuello y hombros, se puede 
asociar a otros síntomas como náuseas, vómito, fotofobia, ceguera, diplopía, acúfenos, sordera, vértigo, somnolencia, parestesias y déficit de pares craneales, empeora en posición erguida por disminución de la PIC y vasodilatación intracraneal y mejora al decúbito supino por equilibro de presiones de LCR en las cisternas intracraneales y el compartimiento lumbar. Su intensidad puede variar desde leve (50\%), moderada (35\%), hasta severa (15\%)[1],[17]-[19].

The International Headache Society (IHS) en 2013, la define como una cefalea que ocurre dentro de los cinco días posteriores a una $\mathrm{PL}$, causada por la fuga de LCR a través de la punción dural, por lo general se acompaña de rigidez en el cuello y/o síntomas subjetivos de la audición y remite espontáneamente dentro de dos semanas o después del sellado de la fuga con parche epidural[20].

Se presenta en un rango del 1 al 70\% de la población implicada, esta gran brecha es el resultado de diferentes técnicas y factores que influencian su incidencia[21], algunos factores de riesgo descritos son: género femenino (relación femenino/masculino 3:1), edad entre 31 y 50 años, historia previa de CPPD, bajo índice de masa corporal (IMC), comorbilidades como síndrome de Marfan y síndrome de Ehlers-Danlos (desórdenes del tejido conectivo), uso de agujas de alto calibre y uso de agujas espinales de bisel cortante (AEBC), más conocidas como agujas espinales traumáticas[2],[22],[23]. Por lo anterior, en 1950, Hart and Whitacre sugirieron el uso de agujas espinales de punta cónica (AEPC) o punta de lápiz, también llamadas agujas espinales atraumáticas, algunas de ellas son: Whitacre, Sprotte y Gertie Marx; La característica crítica de las AEPC es que llevan a pequeño defecto dural, con menor pérdida de LCR y por tanto una incidencia más baja de CPPD. Confirmando dicha teoría, se realizó un estudio en 113 cadáveres a los cuales se les practicó PL con AEBC en 49 de ellos y con AEPC en los 54 restantes, se describe la posterior salida de LCR durante una hora (60 minutos), los resultados arrojan una fuga de LCR de $2.191 \pm 6.146 \mathrm{~mL} / \mathrm{h}$ para AEBC (tipo Quincke) y 0,409 $\pm 0,348 \mathrm{~mL} / \mathrm{h}$ para AEPC (tipo Whitacre)[24],[25].

\section{¿A qué se debe la menor fuga de LCR con las AEPC?}

Este efecto se había atribuido por décadas a que permitía la separación e impedía el corte de las fibras durales, asumiendo que dichas fibras se encontraban dispuestas en forma longitudinal[24],[25], sin embargo, actualmente la microscopía electrónica ha descrito que las fibras de la duramadre están dispuestas aleatoriamente en forma horizontal, longitudinal y oblicua y ha mostrado que las AEPC son más traumáticas que las $A E B C$, ya que produce un desgarro irregular en la duramadre; la menor salida de LCR, entonces, se le atribuye a una reacción inflamatoria subsiguiente que termina en la formación de un "tapón edematoso", la hipótesis se basa en que un trauma contuso presenta mayor edema que un trauma con objeto afilado[26], por lo que resulta contradictorio el nombre de "agujas espinales atraumáticas" que reciben. Contrario a esto, las $A E B C$ se caracterizan por menor reacción inflamatoria, mayor fuga de LCR y por ende mayor incidencia de CPPD[27].

Por lo mencionado, surge la duda de cuan conocida y cuánto uso se le da en el medio hospitalario a la AEPC; una encuesta realizada a 74 neurólogos arroja que el $78 \%$ conocen de ella pero tan solo el $16 \%$ la utilizan de rutina, los motivos fueron: falta de entrenamiento, poca disponibilidad, mayor costo y poca creencia en la evidencia a favor[28].

Debido a que la CPPD es una de las principales complicaciones de la PL y que en la mayoría de los casos es un dolor iatrogénico totalmente innecesario, surge la necesidad de realizar el presente estudio. En este artículo se presenta una revisión de ensayos clínicos aleatorizados, comparando la incidencia de CPPD en la intervención con AEBC y AEPC.

\section{Materiales y Métodos}

Para la realización de esta revisión se recopiló información de estudios clínicos publicados desde el año 2006. La búsqueda de estos artículos se realizó en las bases de datos Pubmed, Science Direct, Clinical Key y EBSCO, con el criterio de identificar estudios aleatorizados que comparen la incidencia de CPPD en AEPC y AEBC, adicionalmente se consultó con expertos en el área que pudieran sugerir literatura relacionada con este tema, la cual no hubiese sido incluída en los métodos de búsqueda previamente descritos. Se utilizaron los términos de búsqueda en inglés para tener una mayor cobertura: whitacre needle, pencil point spinal needle, headache, quincke, quincke needle, post dural puncture headache (PDPH), sprotte; usando conectores booleanos «AND» y «OR». Con los filtros para resultados en español o inglés, solo en humanos y que fueran publicados desde el año 2006. Se excluyeron los artículos duplicados, estudios no aleatorizados, cuyo contenido no compara la incidencia de CPPD en AEPC y AEBC, PL realizada en espacios lumbares diferentes a L2-L3/L3-L4/ L4-L5, inclusión de pacientes menores de 14 años, 
seguimiento del paciente inferior a 5 días posprocedimiento y utilización de aguja espinal con diámetro mayor a 27 G. Además, en la mayoría de los estudios se excluyó a los pacientes con cefalea y migraña recurrente o reciente, con evidencia de aumento de la PIC, pacientes que requirieron 3 o más intentos de PL o PL realizada por personal no experto.

Cada artículo fue evaluado independientemente por 3 investigadores. Las discrepancias se resolvieron por consenso. La evaluación de la calidad metodológica de los estudios aleatorizados se realizó con la herramienta CONSORT, seleccionando para esta revisión aquellos artículos cuyo método fuera transparente y que luego de la calificación, se pudiera asegurar que su calidad es buena, la presentación de estos resultados se hacen en el gráfico de riesgo de sesgo propuesto por la colaboración Cochrane (Figura 1).

\section{Resultados}

Con los criterios de búsqueda se recuperaron 115 artículos, a través de otros recursos se identifica un artículo adicional para un total de 116 artículos, al eliminar los resultados duplicados quedaron 78 artículos. Se realizó primer tamizaje donde se excluyeron 40 artículos cuyo contenido no comparaba la incidencia de CPPD en AEPC y AEBC, posteriormente, se obtuvieron los textos completos y se escogieron aquellos estudios aleatorizados que compararan ambos tipos de agujas en la frecuencia de presentación de cefalea, que la CPPD se midiera según los criterios diagnósticos ICHD-3 beta, que incluyeran pacientes de 14 años en adelante y que se realizara estricto seguimiento de los pacientes posterior al procedimiento por mínimo 5 días. Aplicando estos criterios, se seleccionaron finalmente 14 artículos, los cuales fueron incluídos para la revisión. La Tabla 1 resume los resultados obtenidos con cada una de las estrategias de búsqueda y la Figura 2 el proceso de selección según la guía PRISMA.

Se extrajeron datos de cada uno de los artículos seleccionados de acuerdo con las variables especificadas en la Tabla 2, que incluye: tipo de aguja, diámetro y número de pacientes en el grupo de intervención y en el grupo de comparación, variables de confusión, incidencia de CPPD en ambos grupos, valor de p de la relación entre ambos, Odds ratio e intervalo de confianza $95 \%$.

El tipo de AEBC que se utilizó en todos los estudios fue Quincke, por el contrario, se utilizaron varios tipos de AEPC siendo Sprotte y Whitacre las más prevalentes; el diámetro de las agujas espinales varió

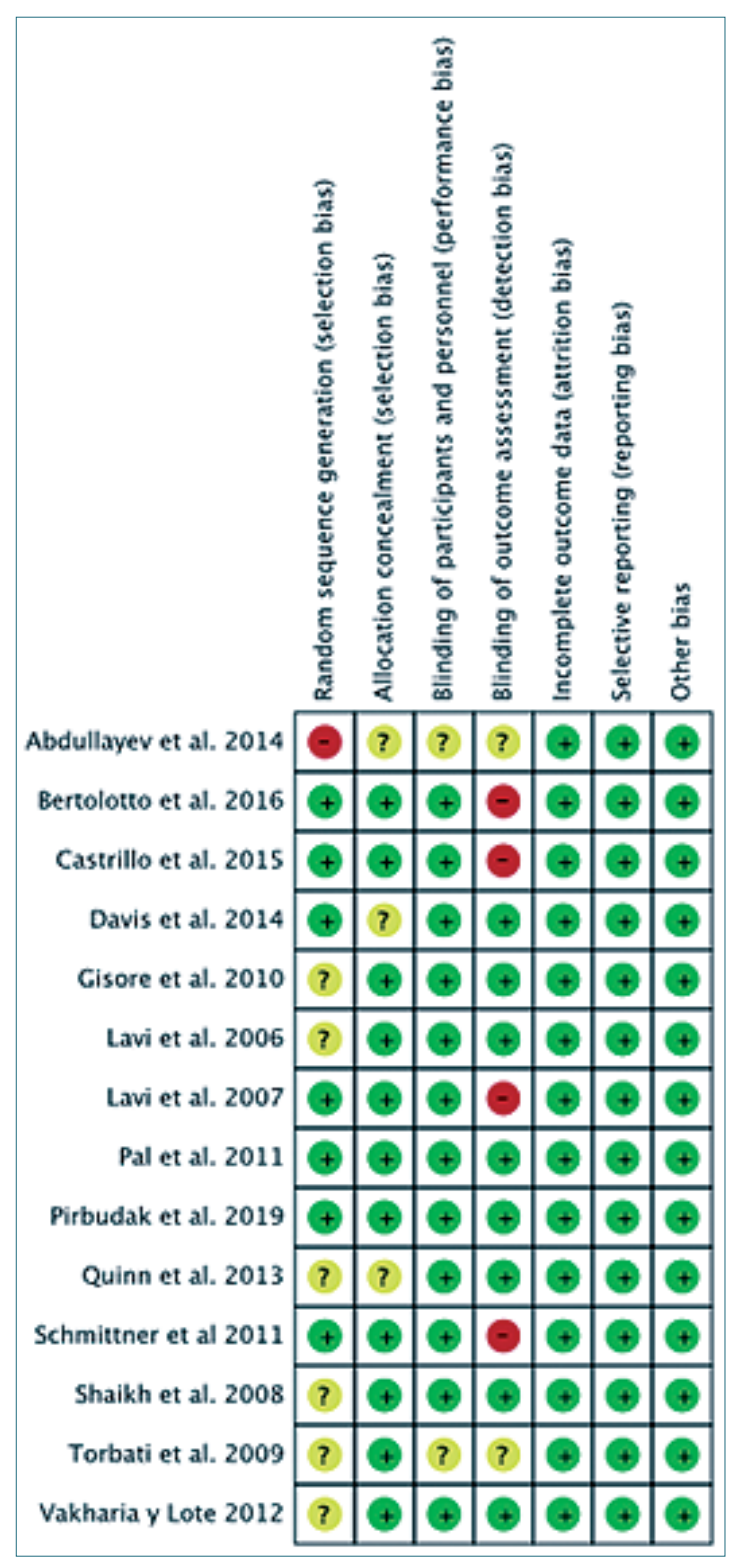

Figura 1. Gráfico de riesgo de sesgo.

entre $20 \mathrm{G}$ hasta $27 \mathrm{G}$, siendo la 22 G la más utilizada (Tabla 2).

Varios estudios tuvieron en cuenta edad, sexo, índice de masa corporal, diagnóstico previo y posición como posibles variables de confusión; en cuanto al sexo, por muchos años la incidencia de CPPD ha sido estimada como 2,5 veces mayor en mujeres que en hombres debido a la elasticidad de las fibras durales[29], Berlotto et al. en 2016[30] fue el único de 
Tabla 1. Resultados de la búsqueda en bases de datos

\begin{tabular}{llc}
\hline Base de datos & Descriptores & Resultados \\
Medline (EBSCO) & PLPH and whitacre needle & 1 \\
& Pencil point spinal needle AND PDPH & 9 \\
& Headache and Whitacre needle AND Quincke & 6 \\
Pubmed & (Headache) and (whitacre needle) & 13 \\
& (Whitacre needle) and (Quincke needle) & 26 \\
Clinical Key & (Pencil point spinal needle) and (post dural puncture headache) & 24 \\
Science Direct & Post dural puncture headache and Whitacre needle & 24 \\
Total & "Post dural puncture headache" and "whitacre needle" & 115 \\
\hline
\end{tabular}

PLPH: Post-lumbar puncture headache, PDPH: Postdural puncture headache.

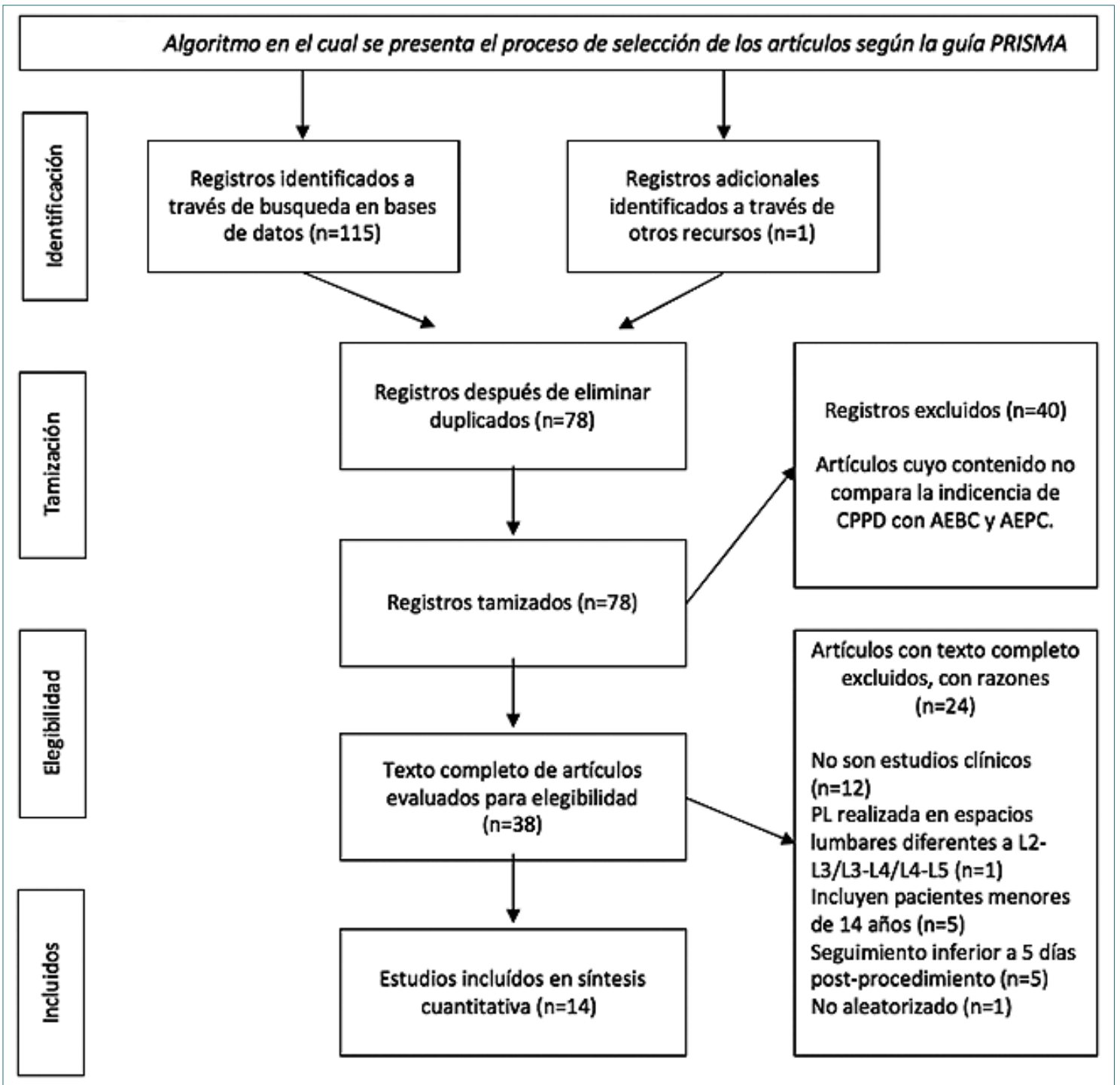

Figura 2. 


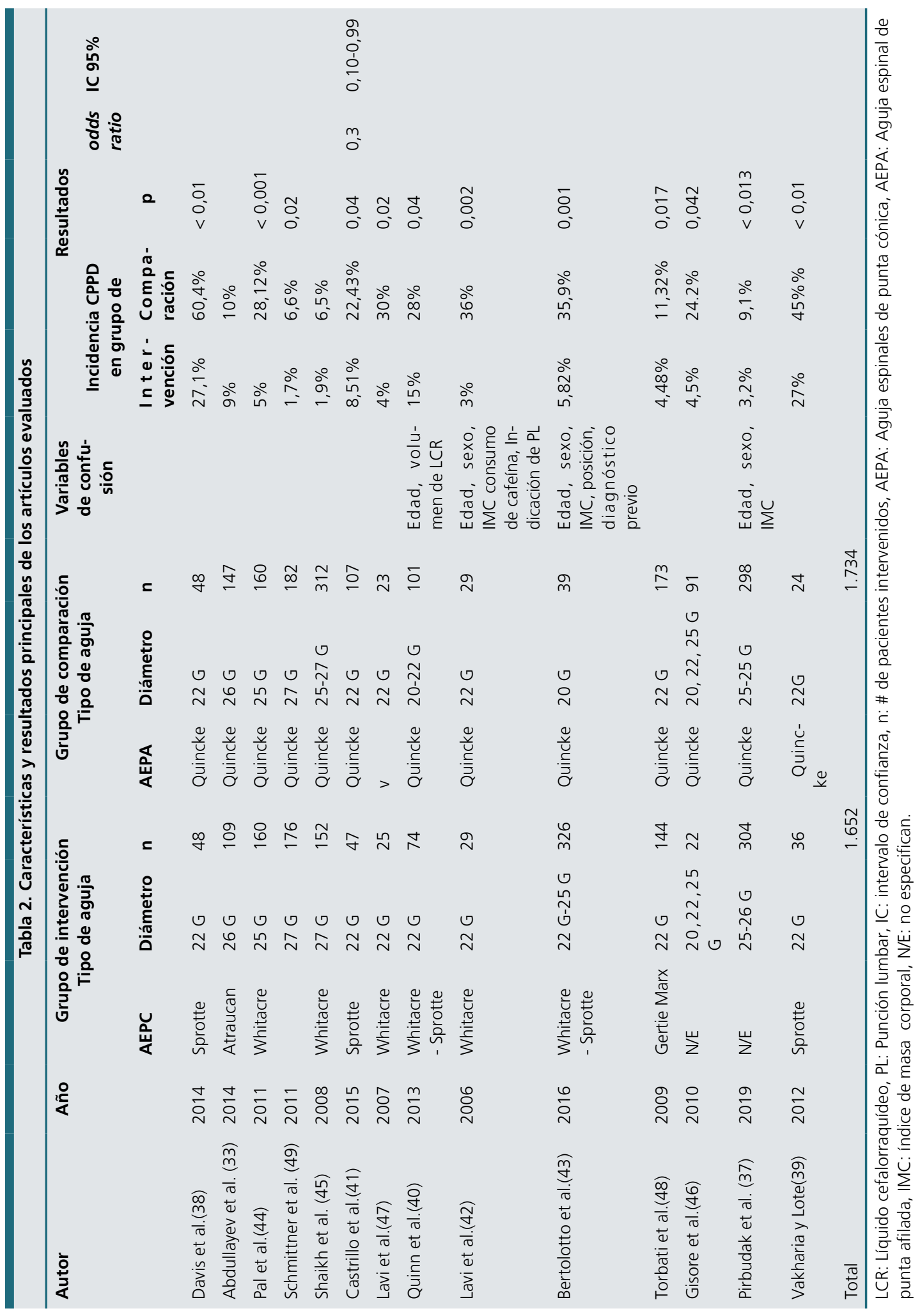


los estudios que no asoció el sexo femenino a mayor riesgo de CPPD, apoyando así otros artículos encontrados[3],[31],[32].

Se recopiló un total de 1.652 pacientes en el grupo de intervención y 1.734 en el grupo de comparación. Todos los artículos evaluados revelaron una menor incidencia de cefalea en los pacientes intervenidos con AEPC, excepto Abdullayev et al. en 2014[33], quienes no encontraron una diferencia estadísticamente significativa en la incidencia de CPPD, siendo además el único estudio que utilizó aguja espinal de tipo Atraucan.

La severidad y duración de la CPPD fue evaluada por 4 estudios, los cuales asocian el uso de AEBC a CPPD de intensidad moderada-severa, de mayor duración y mayor necesidad de analgesia para el cese del cuadro, llegando algunos a requerir parche epidural hemático, por el contrario, relacionan el uso de AEPC a CPPD leve, de menor duración y menor necesidad de analgesia para resolver el cuadro.

En cuanto al número de intentos para lograr una PL exitosa, es evaluado por 7 estudios, 5 de ellos reportan no encontrar ninguna diferencia entre ambas agujas espinales, mientras Castrillo et al. en 2015[34] y Torbati et al. en 2009[35], encuentran un mayor índice de falla con la $A E P C$, requiriendo mayor número de intentos para lograr una adecuada PL.

La incidencia de náuseas y vómito fue evaluada por Pal et al. en 2011[36] pero no encontraron diferencia significativa entre ambas agujas espinales.

Adicionalmente, Pirbudak et al. En 2019[37]"container-title": "Agri: Agri (Algoloji dividieron los pacientes pertenecientes al estudio en 3 grupos, según la edad, para valorar la incidencia de CPPD y encontraron: $18-25$ años $(10,1 \%), 26-40$ años $(8,4 \%)$ y mayores de 40 años $(2,1 \%)$, por lo que fue significativamente menor la incidencia de CPPD en el último grupo $(p<0,008)$.

En los estudios seleccionados se incluyeron pacientes que fueron sometidos a anestesia espinal para cirugía anorrectal, los que requirieron PL terapéutica, especialmente aplicación de quimioterapia intratecal en pacientes con linfoma y leucemia linfoblástica aguda, además pacientes intervenidos para PL diagnóstica en patologías neurológicas como esclerosis múltiple, enfermedades neuroinflamatorias (mielitis transversa, neuromielitis óptica, artritis reumatoide, síndrome de Sjogren, enfermedad de meniere y fibromialgia), hidrocefalia de presión normal incluyendo pseudotumor cerebri, neurosífilis, malignidad e infección (varicela zoster y criptococo) y por último pacientes obstétricas que requerían cesárea bajo anestesia espinal.
Los estudios fueron llevados a cabo en servicios neurológicos[38]-[43], ginecológicos[33],[44]-[46], hematológicos[47], salas de urgencias[48], urológicos[49] e incluso en múltiples servicios[37].

Los resultados detallados de cada uno de los estudios incluidos en esta revisión sistemática, se pueden encontrar en la Tabla 2.

Los artículos incluidos en esta revisión sistemática difieren en cuanto a el diámetro de la aguja espinal utilizada en el grupo de intervención y de comparación, la indicación de la punción y el tipo de cirugía, lo que puede estar reflejando una gran diferencia en la línea basal de estos pacientes al comienzo de cada uno de los ensayos clínicos, dadas estas fuentes de heterogeneidad, no se considera apropiado proseguir con un meta-análisis. ( ${ }^{2}$ : moderado-alto).

\section{Discusión}

Para muchas personas el hecho de ser sometidos a una punción lumbar, es de por sí una experiencia poco agradable, a lo anterior se le suma la CPPD, que en gran porcentaje, es un síntoma evitable y completamente innecesario[28]. El factor predisponente más importante y previamente conocido es el diámetro de la aguja espinal, un menor diámetro se relaciona con menor incidencia de CPPD[50]-[53], adicionalmente, el tipo de aguja espinal es un factor que se asocia a la patogénesis de la CPPD y juega un papel crucial; los resultados de la actual revisión sistemática, en la que se incluyeron 14 estudios clínicos, demostró que la AEPC es la más apropiada para la anestesia espinal y la punción lumbar diagnóstica, por presentar menor incidencia de CPPD, comparado con la AEBC, adicionalmente, en caso de presentarse CPPD, con la AEPC se desarrolla de menor intensidad y corta duración, mientras con $A E B C$ se presenta de mayor intensidad y duración, lo que es explicado por mayor salida de LCR, descenso de las estructuras cerebrales, formación de hematomas subdurales por dilatación del sistema venoso y grandes colecciones de fluidos epidurales que se extienden desde la cauda equina hasta la región torácica inferior, causando presión sobre las raíces nerviosas y desatando dolor[54].

Los hallazgos de la actual revisión sistemática coinciden con otras revisiones sistemáticas, tales como Arévalo-Rodríguez et al.[55] y con los últimos metaanálisis que hay disponible en la literatura[56]-[59], concluyendo que el uso de AEPC es beneficioso para prevenir la aparición de CPPD; no se encuentra en la literatura evidencia a favor de la AEBC.

Basados en la evidencia, ¿Cómo puede introdu- 
cirse la AEPC en la actual cultura de rechazo y evitación?[28].

Se resalta la importancia de la introducción de las AEPC, ya que el uso de intervenciones profilácticas para prevenir la aparición de CPPD, cómo parche epidural hemático, morfina epidural y catéteres intratecales, aún se encuentra con poca evidencia y heterogeneidad en los resultados de los estudios disponibles[29].

Queda claro que una de las principales causas para la no utilización de la AEPC es el mayor costo que algunos especialistas en el tema dicen tener; en un estudio realizado en Stanford, CA. se determina qué tipo de aguja se prefiere desde una perspectiva de costo, arrojando que el uso de la AEPC permite ahorrar $\$ 26,07$ por paciente, lo anterior basado en costos totales posterior al uso de AEPC de $\$ 166,08$, comparado con $\$ 192,15$ en AEBC, teniendo en cuenta no sólo el precio de la aguja sino también lo que implica el manejo de la CPPD, para un total de $\$ 10,4$ millones en ahorro de costos para el sistema de salud de los Estados Unidos de América por año[60]; Otra de las justificaciones para el rechazo de la AEPC es la mayor dificultad en la técnica, por lo que, se necesitan más estudios que analicen la tasa de fallo en un primer intento para lograr una PL exitosa, ya que se encuentran discrepancias en los diferentes estudios y es un factor altamente influenciable por la experiencia del profesional que llevará a cabo el procedimiento; la diferencia más notaria entre ambas técnicas es que con la AEPC se dificulta la introducción, lo cual se soluciona introduciendo una AEBC y una vez que se tiene a $2 \mathrm{~cm}$ de profundidad (depende de la cantidad de tejido adiposo del paciente) se avanza a través de la anterior la AEPC, hasta llegar al espacio subaracnoideo[39].
Se invita así, a la concientización del beneficio que se podría ofrecer a los pacientes con el cambio del tipo de aguja espinal y se sugieren espacios de entrenamiento y capacitación en los diferentes servicios para facilitar dicho cambio.

\section{Conclusión}

Estudios basados en Resonancia Nuclear Magnéti$\mathrm{ca}$, han mostrado que las AEPC son más traumáticas que las $A E B C$, debido a que ocasionan desgarro irregular de la duramadre y mayor respuesta inflamatoria[26], lo que termina en la formación de un "tapón edematoso", cierre del defecto dural, disminución de la fuga de LCR y menor incidencia de CPPD. Según el mecanismo fisiopatológico más recientemente enunciado, resulta contradictorio darle a las AEPC el nombre de "agujas espinales atraumáticas". La actual revisión sistemática, propone un cambio en la nomenclatura que es de mayor utilidad para el anestesiólogo clínico, definiendo AEPC: Withacre, Sprotte y Gertie Marx y AEBC: Quincke. Adicionalmente, la evidencia actual indica que la elección de las AEPC es superior, comparado con las $A E B C$, para la anestesia espinal, PL diagnóstica o terapéutica y punción de la duramadre accidentalmente durante anestesia/analgesia epidural. Lo anterior, debido a que la cefalea postpunción dural se presenta con menor frecuencia, baja intensidad y corta duración en pacientes intervenidos con aguja espinal de punta cónica; se requieren estudios del impacto en la selección del tipo de aguja, para confirmar el ahorro en gastos en el sistema de salud.

\section{Conflicto de intereses}

Ninguno.

\section{Referencias}

1. López Correa T, Garzón Sánchez JC, Sánchez Montero FJ, Muriel Villoria C. Cefalea postpunción dural en obstetricia. Revista Española de Anestesiología y Reanimación [Internet]. Elsevier BV; 2011 Jan;58(9):56373. Available from: http:// dx.doi.org/10.1016/s00349356(11)70141-4

2. Ghaleb A, Khorasani A, Mangar
D. Post-dural puncture headache. Int J Gen Med. 2012;5:4551. https://doi.org/10.2147/ IJGM.S17834 PMID:22287846

3. Lavi R, Rowe JM, Avivi I. Lumbar puncture: it is time to change the needle. Eur Neurol. 2010;64(2):108-13. https:// doi.org/10.1159/000316774 PMID:20628255

4. Grygorczuk S, Pancewicz S, Zajkowska J, Kondrusik M, Hermanowska-Szpakowicz T. [Post- lumbar puncture syndrome--its pathogenesis, prophylaxis and treatment]. Neurol Neurochir Pol. octubre de 2006;40(5):43440.

5. Raskin NH. Lumbar puncture headache: a review. Headache. 1990 Mar;30(4):197-200. https://doi. org/10.1111/j.1526-4610.1990. hed3004197.x PMID:2186014

6. dos Reis A. Eulogy to August Karl Gustav Bier on the 100th 
anniversary of intravenous regional block and the 110 th anniversary of the spinal block. Rev Bras Anestesiol. agosto de 2008;58(4):409-24.

7. Hammond ER, Wang Z, Bhulani N, McArthur JC, Levy M. Needle type and the risk of post-lumbar puncture headache in the outpatient neurology clinic. J Neurol Sci. 15 de julio de 2011;306(12):24-8.

8. Arendt K, Demaerschalk BM, Wingerchuk DM, Camann W. Atraumatic lumbar puncture needles: after all these years, are we still missing the point? The Neurologist. enero de 2009;15(1):1720. https://doi.org/10.1097/ NRL.0b013e318184f476.

9. Butterworth JF 4th, Mackey DC, Wasnick JD. The Practice of Anesthesiology. En: Morgan \& Mikhail's Clinical Anesthesiology [Internet]. 6.a ed. New York, NY: McGraw-Hill Education; 2018 [citado 22 de mayo de 2019]. Disponible en: accessmedicine.mhmedical.com/content. aspx?aid=1161425336

10. A. D. Doyle A. Anesthesia. Choices and Complications. En: McKean SC, Ross JJ, Dressler DD, Scheurer DB, editores. Principles and Practice of Hospital Medicine [Internet]. 2.a ed. New York, NY: McGraw-Hill Education; 2017 [citado 22 de mayo de 2019]. Disponible en: accessmedicine.mhmedical.com/ content. aspx?aid=1137607863

11. Quintero IF, Candamil Á, Mejía Mantilla JH, Medina H, Ariza Cadena F. Síndrome de hipotensión endocraneana: ¿una cefalea pospunción dural? Rev Colomb Anestesiol. marzo de 2013;41(1):57-60.

12. Lay CM. Low Cerebrospinal Fluid Pressure Headache. Curr Treat Options Neurol. septiembre de 2002;4(5):357-63. https://doi. org/10.1007/s11940-002-00469.

13. Miyazawa $K$, Shiga $Y$, Hasegawa T, Endoh M, Okita N, Higano S, et al. CSF hypovolemia vs intracranial hypotension in «spontaneous intracranial hypotension syndrome». Neurology. 25 de marzo de 2003;60(6):941-7.

14. Booth JL, Pan PH, Thomas JA, Harris LC, D'Angelo R. A retrospective review of an epidural blood patch database: the incidence of epidural blood patch associated with obstetric neuraxial anesthetic techniques and the effect of blood volume on efficacy. Int J Obstet Anesth. febrero de 2017;29:10-7. https://doi.org/10.1097/01. aoa.0000527038.75691.19.

15. Tien JC, Lim MJ, Leong WL, Lew E. Nine-year audit of post-dural puncture headache in a tertiary obstetric hospital in Singapore. Int J Obstet Anesth. diciembre de 2016;28:34-8. https://doi. org/10.1016/j.ijoa.2016.06.005.

16. Sandesc D, Lupei MI, Sirbu C, Plavat C, Bedreag O, Vernic C. Conventional treatment or epidural blood patch for the treatment of different etiologies of post dural puncture headache. Acta Anaesthesiol Belg. 2005;56(3):265-9. PMID:16265829

17. Wu C, Lian Y, Xie N. Aminophylline Injection Alleviates Pain in Postdural Puncture Headache. Pain Med Malden Mass. octubre de 2015;16(10):203840. https://doi.org/10.1111/ pme. 12804

18. Park KM, Shin KJ, Ha SY, Park J, Kim SE. Does lumbar puncture at night prevent postdural puncture headache? Acta Neurol Scand. septiembre de 2014;130(3):204-9. https://doi. org/10.1111/ane.12267.

19. Fattahi Z, Hadavi SMR, Sahmeddini MA. Effect of ondanse- tron on post-dural puncture headache (PDPH) in parturients undergoing cesarean section: a double-blind randomized placebo-controlled study. J Anesth. octubre de 2015;29(5):702-7.

20. The International Classification of Headache Disorders, 3rd edition (beta version). Cephalalgia [Internet]. SAGE Publications; 2013 Jun 14;33(9):629808. Available from: http://x. doi.1177/0333102413485658.

21. Toyka KV, Müller B, Reichmann $\mathrm{H}$. «Atraumatic» Sprotte needle reduces the incidence of postlumbar puncture headaches. Neurology. 8 de octubre de 2002;59(7):1120; author reply 1120-1121. https://doi. org/10.1212/WNL.59.7.1120.

22. Lotfy Mohammed E, El Shal SM. Efficacy of different size Quincke spinal needles in reduction of incidence of Post-Dural Puncture Headache (PDPH) in Caesarean Section (CS). Randomized controlled study. Egypt J Anaesth. 1 de enero de 2017;33(1):53-8.

23. Vidoni ED, Morris JK, Raider K, Burns JM, Alzheimer's Disease Neuroimaging Initiative. Reducing post-lumbar puncture headaches with small bore atraumatic needles. J Clin Neurosci Off J Neurosurg Soc Australas. marzo de 2014;21(3):536-7.

24. Yumpu.com. Complicaciones de la Anestesia locorregional [Internet]. yumpu.com. [citado 22 de mayo de 2019]. Disponible en: https://www.yumpu.com/ es/document/read/17201288/ complicaciones-de-la-anestesialocorregional

25. Jabbari A, Hasanjani Roushan MR. Impact of postdural puncture headache after diagnostic lumbar puncture. Caspian J Intern Med. 2014;5(2):56-8. PMID:24778777

26. Reina MA, de Leon-Casasola OA, López A, De Andrés J, 
Martin S, Mora M. An in vitro study of dural lesions produced by 25-gauge Quincke and Whitacre needles evaluated by scanning electron microscopy. Reg Anesth Pain Med. agosto de 2000;25(4):393-402.

27. García Rodríguez A, Martínez Mompeller O, Rodríguez $\mathrm{P}$, Luis M, Correa Torres M, Mirabal $M$, et al. Cefalea secundaria a punción dural. Rev Cienc Médicas Pinar Río. marzo de 2005;9(1):51-60.

28. Davis A, Dobson R, Kaninia $S$, Espasandin M, Berg A, Giovannoni $G$, et al. Change practice now! Using atraumatic needles to prevent post lumbar puncture headache. Eur J Neurol. febrero de 2014;21(2):305-11. https:// doi.org/10.1111/ene.12307.

29. Reynolds F. Dural puncture and headache. BMJ. 3 de abril de 1993;306(6882):8746. https://doi.org/10.1136/ bmj.306.6882.874.

30. Bertolotto A, Malentacchi M, Capobianco M, di Sapio A, Malucchi S, Motuzova Y, et al. The use of the 25 Sprotte needle markedly reduces post-dural puncture headache in routine neurological practice. Cephalalgia Int J Headache. febrero de 2016;36(2):131-8.

31. Amorim JA, Gomes de Barros MV, Valença MM. Postdural (post-lumbar) puncture headache: risk factors and clinical features. Cephalalgia Int J Headache. septiembre de 2012;32(12):916-23.

32. Bezov D, Ashina S, Lipton R. Post-dural puncture headache: Part II--prevention, management, and prognosis. Headache. octubre de 2010;50(9):1482-98.

33. Comparison of Two Spinal Needles Regarding Postdural Puncture Headache - Study Results - ClinicalTrials. gov [Internet]. [citado 29 de abril de
2019]. Disponible en: https:// clinicaltrials.gov/ct2/show/results/NCT01821807

34. Castrillo A, Tabernero $C$, García-Olmos LM, Gil C, Gutiérrez R, Zamora Ml, et al. Postdural puncture headache: impact of needle type, a randomized trial. Spine J. 1 de julio de 2015;15(7):15716. https://doi.org/10.1016/j. spinee.2015.03.009.

35. Torbati S, Katz D, Silka P, Younessi S. 234: Comparison of Blunt Versus Sharp Spinal Needles Used in the Emergency Department in Rates of PostLumbar Puncture Headache. Ann Emerg Med. 1 de septiembre de 2009;54(3):S73.

36. Pal A, Acharya A, Pal ND, Dawn S, Biswas J. Do pencilpoint spinal needles decrease the incidence of postdural puncture headache in reality? A comparative study between pencil-point 25G Whitacre and cutting-beveled 25G Quincke spinal needles in 320 obstetric patients. Anesth Essays Res. julio de 2011;5(2):162-6.

37. Pirbudak L, Özcan HI, Tümtürk P. Postdural puncture headache: incidence and predisposing factors in a university hospital. Agri. 2019 Jan;31(1):1-8. PMID:30633317

38. Davis A, Dobson R, Kaninia S, Espasandin M, Berg A, Giovannoni $\mathrm{G}$, et al. Change practice now! Using atraumatic needles to prevent post lumbar puncture headache. Eur J Neurol. febrero de 2014;21(2):305-11. https:// doi.org/10.1111/ene.12307.

39. Vakharia VN, Lote H. Introduction of Sprotte needles to a single-centre acute neurology service: before and after study. JRSM Short Rep [Internet]. 15 de diciembre de 2012 [citado 29 de abril de 2019];3(12). Disponible en: https://www.ncbi.nlm.nih.
gov/pmc/articles/PMC3545339/

https://doi.org/10.1258/

shorts.2012.012090.

40. Quinn C, Macklin EA, Atassi N, Bowser R, Boylan K, Cudkowicz $M$, et al. Post-lumbar puncture headache is reduced with use of atraumatic needles in ALS. Amyotroph Lateral Scler Front Degener. 1 de diciembre de 2013;14(7-8):632-4. https://doi. org/10.3109/21678421.2013.80 8227.

41. Castrillo A, Tabernero $C$, García-Olmos LM, Gil C, Gutiérrez R, Zamora Ml, et al. Postdural puncture headache: impact of needle type, a randomized trial. Spine J. julio de 2015;15(7):1571-6. https://doi.org/10.1016/j. spinee.2015.03.009.

42. Lavi R, Yernitzky D, Rowe JM, Weissman A, Segal D, Avivi I. Standard vs atraumatic Whitacre needle for diagnostic lumbar puncture: A randomized trial. [Miscellaneous Article]. Neurology. octubre de 2006;67(8):14924.

43. Bertolotto $A$, Malentacchi $M$, Capobianco M, di Sapio A, Malucchi S, Motuzova $Y$, et al. The use of the 25 Sprotte needle markedly reduces postdural puncture headache in routine neurological practice. Cephalalgia. 1 de febrero de 2016;36(2):131-8.

44. Pal A, Acharya A, Pal ND, Dawn S, Biswas J. Do pencil-point spinal needles decrease the incidence of postdural puncture headache in reality? A comparative study between pencil-point $25 G$ Whitacre and cuttingbeveled 25G Quincke spinal needles in 320 obstetric patients. Anesth Essays Res. 2011 Jul-Dec;5(2):162-6. https://doi. org/10.4103/0259-1162.94757 PMID:25885381

45. Shaikh JM, Memon A, Memon 
MA, Khan M. Post dural puncture headache after spinal anaesthesia for caesarean section: a comparison of $25 \mathrm{~g}$ Quincke, $27 \mathrm{~g}$ Quincke and $27 \mathrm{~g}$ Whitacre spinal needles. J Ayub Med Coll Abbottabad JAMC. septiembre de 2008;20(3):10-3.

46. Gisore E, Mung'ayi $V$, Sharif T. Incidence of post dural puncture headache following caesarean section under spinal anaesthesia at the Aga Khan University Hospital, Nairobi. East Afr Med J. junio de 2010;87(6):227-30.

47. Lavi R, Rowe JM, Avivi I. Traumatic vs. atraumatic $22 \mathrm{G}$ needle for therapeutic and diagnostic lumbar puncture in the hematologic patient: a prospective clinical trial. Haematologica. 1 de julio de 2007;92(7):1007-8.

48. Torbati S, Katz D, Silka P, Younessi S. 234: Comparison of Blunt Versus Sharp Spinal Needles Used in the Emergency Department in Rates of PostLumbar Puncture Headache. Ann Emerg Med. 1 de septiembre de 2009;54(3):S73.

49. Schmittner MD, Urban $N$, Janke A, Weiss C, Bussen DG, Burmeister MA, et al. Influence of the pre-operative time in upright sitting position and the needle type on the incidence of postdural puncture headache (PDPH) in patients receiving a spinal saddle block for anorectal surgery. Int J Colorectal Dis. enero de 2011;26(1):97-102.

50. Bezov D, Lipton RB, Ashina S.
Post-dural puncture headache: part I diagnosis, epidemiology, etiology, and pathophysiology. Headache. julio de 2010;50(7):1144-52. https:// doi.org/10.1111/j.15264610.2010.01699.x.

51. Flaatten $H$, Thorsen $T$, Askeland $B$, Finne M, Rosland J, Hansen $\mathrm{T}$, et al. Puncture technique and postural postdural puncture headache. A randomised, double-blind study comparing transverse and parallel puncture. Acta Anaesthesiol Scand. noviembre de 1998;42(10):1209-14.

52. Apiliogullari S, Duman A, Gok F, Akillioglu I. Spinal needle design and size affect the incidence of postdural puncture headache in children. Paediatr Anaesth. febrero de 2010;20(2):177-82. https://doi.org/10.1111/j.14609592.2009.03236.x.

53. O'Connor G, Gingrich R, Moffat $M$. The effect of spinal needle design, size, and penetration angle on dural puncture cerebral spinal fluid loss. AANA J. abril de 2007;75(2):111-6.

54. Lavi R, Rowe JM, Avivi I. Lumbar Puncture: It Is Time to Change the Needle. Eur Neurol. 5 de agosto de 2010;64(2):108-13.

55. Arevalo-Rodríguez I, Muñoz L, Godoy-Casasbuenas N, Ciapponi A, Arevalo JJ, Boogaard S, et al. Needle gauge and tip designs for preventing post-dural puncture headache (PDPH). Cochrane Database Syst Rev. 7 de abril de 2017;4:CD010807.
56. Xu H, Liu Y, Song W, Kan S, Liu F, Zhang D, et al. Comparison of cutting and pencil-point spinal needle in spinal anesthesia regarding postdural puncture headache: A meta-analysis. Medicine (Baltimore). abril de 2017;96(14):e6527-e6527. https://doi.org/10.1097/ MD.0000000000006527.

57. Lee SI, Sandhu S, Djulbegovic B, Mhaskar RS. Impact of spinal needle type on postdural puncture headache among women undergoing Cesarean section surgery under spinal anesthesia: A meta-analysis. J Evid-Based Med. agosto de 2018;11(3):136-44.

58. Zorrilla-Vaca A, Mathur V, Wu $\mathrm{CL}$, Grant MC. The Impact of Spinal Needle Selection on Postdural Puncture Headache: A Meta-Analysis and Metaregression of Randomized Studies. Reg Anesth Pain Med. julio de 2018;43(5):5028. https://doi.org/10.1097/ AAP.0000000000000775.

59. Zhang $D$, Chen L, Chen $X$, Wang $X$, Li $Y$, Ning G, et al. Lower incidence of postdural puncture headache using whitacre spinal needles after spinal anesthesia: A meta-analysis. Headache. marzo de 2016;56(3):50110. https://doi.org/10.1111/ head. 12745.

60. Tung CE, So YT, Lansberg MG. Cost comparison between the atraumatic and cutting lumbar puncture needles. Neurology. 10 de enero de 2012;78(2):109-13. 